(To appear in: Journal of Marine Systems

special issue on Marine Rapid Environmental Assessment

Submitted Nov 23, 2005, revised May 17, 2006, accepted May 19, 2006)

\title{
Collaboration Tools and Techniques for Large Model Datasets
}

\author{
Richard P. Signell $^{\mathrm{a}, *}$, Sandro Carniel ${ }^{\mathrm{b}}$, Jacopo Chiggiato ${ }^{\mathrm{c}}$, Ivica Janekovic ${ }^{\mathrm{d}}$, \\ Julie D. Pullen ${ }^{\mathrm{e}}$, and Christopher R. Sherwood ${ }^{\mathrm{f}}$ \\ ${ }^{a}$ NATO Undersea Research Centre, Viale San Bartolomeo 400, 19138 La Spezia, Italy, (now at U.S. \\ Geological Survey, 384 Woods Hole Rd, Woods Hole, MA 02543 USA) \\ ${ }^{b}$ Institute of Marine Sciences (ISMAR), National Research Council, San Polo 1364, 30125 Venice, Italy \\ ${ }^{c}$ Servizio IdroMeteorologico-ARPA Emilia Romagna, Viale Silvani 6, 40122 Bologna, Italy \\ ${ }^{d}$ Rudjer Boskovic Institute, Center for Marine and Environmental Research, Bijenicka C54, 10000 Zagreb, \\ Croatia \\ ${ }^{e}$ Naval Research Laboratory, 7 Grace Hopper Rd, Monterey, CA, 93943 USA \\ ${ }^{f}$ U.S. Geological Survey, 384 Woods Hole Rd, Woods Hole, MA 02543 USA
}

\begin{abstract}
In MREA and many other marine applications, it is common to have multiple models running with different grids, run by different institutions. Techniques and tools are described for low-bandwidth delivery of data from large multidimensional data sets, such as those from meteorological and oceanographic models, directly into generic analysis and visualization tools. Output is stored using the NetCDF CF Metadata Conventions, and then delivered to collaborators over the web via OPeNDAP.

OPeNDAP datasets served by different institutions are then organized via THREDDS catalogs. Tools and procedures are then used which enable scientists to explore data on the original model grids using tools they are familiar with. It is also low-bandwidth, enabling users to extract just the data they require, an important feature for access from ship or remote areas. The entire implementation is simple enough to be handled by modelers working with their webmasters - no advanced programming support is necessary.

Abbreviations: MREA; Marine Rapid Environmental Assessment, NetCDF; Network Common Data Format, CF; Climate and Forecast, OPeNDAP; Open-source Project for Network Data Access Protocol, THREDDS; Thematic Real time Environmental Distributed Data Services.
\end{abstract}

Keywords: Data collections, Information systems, Modelling, Adriatic Sea.

\section{Introduction}

In the field of Marine Rapid Environmental Assessment (MREA) it is now common to have multiple numerical models running in the same oceanic region, all producing large amounts of data on different grids (Coelho, 2006; Onken et al., 2005; Rixen, 2006; Signell et al., 2005). While conventional server side packaging of information and Geographic Information System (GIS) delivery are usually the appropriate methods for

\footnotetext{
* Corresponding author. Tel.: +1-508-548-8700; fax: +1-508-457-2310

E-mail address: rsignell@usgs.gov
} 
delivery to the end users (Kantha et al., 2002), scientists seeking to assess and improve the system need direct, efficient access to the raw data products produced by the models. We describe here a method that was developed out of practical necessity during a large multi-institutional sea trial in the Adriatic Sea that took place from 2002-2003 (Lee et al., 2005; Sherwood et al., 2004), but the method could be applied to any collaborative project involving multiple earth systems models, from global climate change to coastal observing systems, to near-shore littoral field trials.

Numerical weather and ocean models typically produce large four-dimensional data sets that range from tens of MB to many GB; often this information is delivered to the intended "customer" via the web as graphical images from static collections, or increasingly, from dynamic Open GIS Consortium (OGC) servers. While delivery of actual data through OGC servers is possible, currently most OGC servers supply images created from the data via Web Map Service (WMS). Scientists usually want to obtain the actual data, or at the very least, be able to explore the data exactly the way they want to, using their own analysis and visualization tools. They typically do not need all the data. They are usually interested in certain variables at certain times or in certain regions and need an efficient way to slice and dice these data sets over the web. They also do not want to learn a new set of tools for each different model, and would like a consistent interface that can access any model output without regard to how the original model output was written or what vertical or horizontal coordinate system was used (Figure 1).

Constructing general clients that can work with many different types of model products requires standardization, and this means conventions that can represent the spatial representation of model information. One form of standardization is to require that model products be produced on regular longitude/latitude grids with fixed vertical levels. Indeed this is one form of standardization - standardizing the output. But a much more powerful way is standardizing the specification of how model information is encoded in the output files. This is much more appealing to scientists, as they want to access the model output in a form as close as possible to the original model output so that the scientific content of the output is maintained. For example, if they are interested in exploring how well a particular model performs very close to the surface of the ocean, they don't want to find that the many near-surface following layers have been interpolated onto a few fixed standard levels chosen to facilitate data distribution.

Fortunately there are emerging software tools, techniques and standards that make it easy to deliver model output efficiently over the web. One collection of techniques will be described here, as developed through a practical effort (largely by scientists) to effectively share meteorological, wave and circulation model output results within a large multi-institutional international project in the Adriatic Sea.

\section{Lessons from the Adriatic Sea: A Recipe for Sharing Model Output}

\subsection{Store data in a machine-independent, self-describing format}

A fundamental component for effective collaboration is to save model results in a form that is machine independent, binary and self-describing. There are several formats that meet these criteria and are in wide use in the earth sciences community: NetCDF 
(Network Common Data Form), HDF (Hierarchical Data Format), and GRIB (Gridded Binary) are arguably the most popular. We used NetCDF (UNIDATA, 2006b) due to its relative simplicity (less than 30 function calls) and widespread use in the oceanographic community. It is freely available, is supported by Unidata, and has interfaces for many languages, including FORTRAN, C, C++, Java, Perl, Matlab, and IDL. NetCDF allows metadata to be provided both for specific variables in the file and for the entire dataset in the form of variable or global attributes. There is no limit on the number of attributes or the length of any attribute in NetCDF. There can be a character attribute, for example, that is the entire text of the user's manual. Yet there is also no requirement for attributes imposed by NetCDF itself. It is perfectly valid to write a NetCDF file without any attributes. In this case the data types and size of arrays are still present, so it will be possible to read the file accurately, but users may not know what they are looking at.

Although we used NetCDF, it is not so important which specific format is used, because all these formats, if supplied with sufficient metadata, can be represented by a common data model delivered through the web, as discussed below. In fact, the NetCDF version 4 API will actually write HDF files, which may also accessed via the HDF API.

\subsection{Use the Climate and Forecast (CF) Conventions}

One of the strong points of NetCDF is that it places few demands on the data provider they are free to specify whatever attributes they want, or none at all. This, however, is also a weak point, making it difficult to develop clients that can perform useful higher level functions on general NetCDF files. For example, it is hard to make a geographical browser client for ocean model data if it is not known what the independent and dependent variables are, what the units are, etc. The consequence is that even though many ocean models write NetCDF output, they typically use different conventions. This means that software built for the ocean model ROMS, for example, does not work for the ocean models POM, HOPS, NCOM, HYCOM or Delft3D, even though these models all use orthogonal curvilinear coordinates in the horizontal, and have a fixed number of layers in the vertical.

To address this issue, the community has come up with various conventions for specifying metadata in geophysical models. The convention we used was the NetCDF Climate and Forecast Metadata Convention, version 1.0 (Eaton et al., 2003). The goal of CF is to build upon the success of COARDS (Cooperative Ocean/Atmosphere Research Data Service), the first convention in widespread use that provided a consistent specification of longitude, latitude, depth and time. While COARDS restricted longitude, latitude, depth and time to be 1D arrays, CF is much more flexible, and allows for specification of 2D longitude and latitude variables through the use of the "coordinates" attribute, and for specification of formulae to be used for on-the-fly calculation of vertical coordinates via the "standard_name" and "formula_terms" attributes. For example, for output stored in sigma coordinates, the vertical position at a certain time is described by a formula such as

$z(n, k, j, i)=e t a(n, j, i)+\operatorname{sigma}(k) *(\operatorname{depth}(j, i)+e t a(n, j, i))$

where $z(n, k, j, i)$ is height, positive upwards, relative to ocean datum (e.g. mean sea level) 
at grid point $(n, k, j, i)$, eta( $n, j, i)$ is the height of the ocean surface, positive upwards, relative to ocean datum at grid point $(n, j, i), \operatorname{sigma}(k)$ is the dimensionless coordinate at vertical grid point $(k)$, and $\operatorname{depth}(j, i)$ is the distance from ocean datum to sea floor (positive value) at horizontal grid point $(j, i)$.

A CF compliant file would then have a standard name attribute with the value "ocean_sigma_coordinate" and identify which variable names correspond to the terms in the above equation by use of the "formula_terms" attribute. Figure 2 shows the metadata for CF-compliant output from a curvilinear, sigma-coordinate ocean model.

CF 1.0 understands the following vertical dimensionless ocean model coordinates: “ocean_sigma_coordinate”, “ocean_s_coordinate”, “ocean_sigma_z_coordinate”, "ocean_double_sigma_coordinate". One issue that was not made clear in CF 1.0 was whether a sigma variable could be $3 \mathrm{D}(\mathrm{x}, \mathrm{y}, \mathrm{z})$ or $4 \mathrm{D}(\mathrm{x}, \mathrm{y}, \mathrm{z}, \mathrm{t})$ instead of simply $1 \mathrm{D}(\mathrm{z})$. If this were allowed, CF could also accommodate models like HYCOM (Hybrid Coordinate Ocean Model), where some of the layers could be following isopycnals, and therefore changing in space and time.

The example above also shows the CF convention for identifying the horizontal coordinate variables. For each dependent variable a "coordinates" attribute can be specified that simply lists the independent coordinate variables. In the case above, the horizontal coordinates for temperature are the 2D arrays storing the latitude and longitude ("lat" and "lon"). Further conventions are required to determine the type of coordinate represented by these variables. For example, latitude, longitude and time coordinates are identified by their "units" attribute. See the CF specification for full details (Eaton et al, 2003).

Note also that time is referenced to midnight UTC on a fixed Gregorian date so that the time data may be unambiguously understood. Referencing time to a Gregorian date that is before the Gregorian calendar was adopted, such as 1-1-1 00:00 can lead to confusion, and is therefore not recommended. Time in days since 1858-11-17 00:00 is convenient since the time values are then recognized as Modified Julian Day (MJD), a convention introduced by space scientists in the 1950's and sanctioned by several international organizations (IAU, 1997). Note that MJD starts at midnight, which is often more convenient than the astronomical Julian Day (which starts at noon), and are relative to 00:00 on November 17, 1858, a Gregorian date that occurred after most of the world had adopted today's Gregorian calendar.

To confirm that output files are truly CF-compliant, they can be checked by using the CFChecker web form at the British Atmospheric Data Centre: http://titania.badc.rl.ac.uk/cgibin/cf-checker.pl). This is a particularly valuable tool in the early stages of trying to generate CF-compliant data, as the standard is somewhat complex, and clients to read CF-compliant data do not always give helpful error messages to say why they have failed.

CF 1.0 is a large step toward allowing full specification of model grid information in ocean models, yet there is still work to be done. For example, CF 1.0 has no method for specifying more sophisticated connections between grid elements such as those that exist 
in unstructured grids or mosaics of grids. It also does not provide a convention for efficient handling of staggered grids, such as the commonly used Arakawa $\mathrm{C}$ grid. On such a grid, the " $u$ " and " $v$ " points for the horizontal velocity components do not coincide with the "eta" points for the free surface. Thus the formula for the calculation for the vertical coordinate at these locations often cannot be determined without interpolation of the free surface from the surrounding points. For the sigma coordinate model on a $\mathrm{C}$ grid, for example, a CF compliant client would need to determine points nearby and perform a general interpolation of "eta" to the " $u$ " and " $v$ " points. If it was known that this was a $\mathrm{C}$ grid, the client could simply average the neighboring 2 "eta" points that bracket the specific " $u$ " or " $v$ " point, a much more efficient operation. The specification of more complex grid relationships has been proposed for the next release of CF. There is also active discussion of conventions to specify full georeferencing information that would allow CF data compliant data to interface with GIS software. To stay abreast of recent developments or to suggest improvements for $\mathrm{CF}$, one can participate in the CF discussion list.

\subsection{Use and develop generic visualization and analysis tools that work with CF- compliant data}

The promise of CF is to allow clients to be written that can work with any ocean model output, provided that the model output is CF compliant. In the near future there could be CF-compliant toolkits for specific environments like Matlab and IDL, as well as freely-available stand-alone packages written in extensible languages such as Python or Java.

An example of this type of client is the Integrated Data Viewer (IDV) being developed by the Unidata Program Center (UNIDATA, 2006a). This client provides 1D and 2D slicing, 3D rendering, animation, and much more. It is written completely in Java, and can run on any platform that supports Java3D (e.g. Windows, Mac, Linux, and many Unix machines). IDV version 1.2 supports “ocean_sigma_coordinate” and "ocean_s_coordinate" as well as a number of atmospheric vertical coordinates. (Support for all the CF vertical coordinate representations is high on the priority list for development.) The IDV is therefore already capable of displaying results from models like POM or Delft3D together with results from models such as ROMS. It can also perform operations on extracted information via Jython (Python implemented in Java) scripts. These operations can be simple linear transformations, more complex transformation such as the computation of the Richardson Number from several variables, or however complicated a function the user can write in Python. It is therefore easy for end-users to extend or tailor IDV functionality to their own applications and to contribute routines to an ever-growing pool of IDV functionality. 
In the Adriatic Sea work, we used the IDV to simultaneously display CF-compliant meteorological, wave and ocean model results. For example, Figure 1 shows wind vectors from COAMPS ${ }^{\circledR}$ meteorological model superimposed on bottom sediment concentrations from the coupled hydrodynamic and sediment model ROMS during a strong wind event. The IDV does not have to know that this is output from ROMS, COAMPS ${ }^{\circledR}$ or any other specific model, only that the data is CF-compliant. While IDV can read local CF-compliant NetCDF files, it can also read NetCDF files that have been placed on a remote web site. It can also read GeoTIFF images, ESRI Shapefiles, data delivered via OPeNDAP (see next section), and an increasing number of other formats.

The IDV is an excellent "reference application" for CF, and it is extremely useful that it is evolving as the CF standard is evolving. For example, the previously mentioned need to evolve the standards to handle staggered grids with dimensionless vertical coordinates was discovered with we attempted to use the IDV to create a vertical slice of the eastward velocity component. The simultaneous development of reference applications and standards fosters maximization of utility and minimization of useless complexity.

\subsection{Use OPeNDAP to distribute CF-compliant files}

Once the model output is CF-compliant, it can be distributed to others via the web. When CF-compliant web files are simply placed on a web-server accessible directory they become accessible to several clients (such as the IDV) that can extract information and slices of data from remote NetCDF files. There are many more clients, however, that can access data via OPeNDAP (Open-source Project for Network Data Access Protocol), which makes locally-served data accessible to remote locations regardless of local storage format (http://www.OPeNDAP.org/). OPeNDAP was formerly called DODS (Distributed Ocean Data System) and was developed specifically for dealing with efficient distribution of multi-dimensional scientific data sets over the web. It is mainly a collection of servers and clients, which can be used together to serve and access OPeNDAP data, but it also contains libraries ( $\mathrm{C}++$, Java, Fortran) that can be used to turn existing applications into OPeNDAP clients. OPeNDAP can serve not only NetCDF files, but many other common scientific data formats, including HDF (Hierarchical Data Format), Matlab and GRIB (Gridded Binary) files.

An important characteristic of OPeNDAP is that it is very straightforward to install and get running. The server executables are downloaded for the intended operating system and placed in the web server's cgi-bin directory. The model output files (in any of the supported formats) are then placed in a directory that is web-accessible, a configuration file is modified, and OPeNDAP data is being served. It took us less than 1 hour to start serving OPeNDAP data.

OPeNDAP data can be accessed via many methods. There are stand-alone clients that can browse and extract data, and there are also interfaces to many common analysis and visualization environments (Matlab, IDL and Python, Perl, Java-based tools). As an example of turning an existing tool into an OPeNDAP tool, we took the existing MatlabNetCDF interface "mexnc" and recompiled it with the OPeNDAP NetCDF wrapper 
library instead of the standard NetCDF library. The result was an OPeNDAP-enabled "mexnc" Matlab tool (available at http://mexcdf.sourceforge.net/) that functioned as before, but instead of only working with local NetCDF files, could work with data from OPeNDAP server.

Figure 3 shows a snippet of Matlab code utilizing the NetCDF toolbox with underlying "OPeNDAP-enabled mexnc" to access and visualize the $\mathrm{M}_{2}$ major axis current magnitude from an unstructured mesh tidal model of the Adriatic Sea (Janekovic and Kuzmic, 2005). Using OPeNDAP, less than $1 \mathrm{Mb}$ of data is extracted from this remote $1600 \mathrm{Mb}$ file, taking only seconds (14 seconds on a notebook pc connected to the internet via a DSL line). And the data is delivered directly into Matlab, bypassing the cumbersome conversion that Matlab users typically need to apply to data downloaded via the internet.

\subsection{Use THREDDS to catalog distributed data sets}

Though it is easy to serve data sets via OPeNDAP, it may not be easy for users to find out exactly what data are being shared by various institutions. One simple way to do this is to list the datasets in a THREDDS (Thematic Realtime Environmental Distributed Data Services) catalog (UNIDATA, 2006c). A THREDDS catalog in its most primitive form is simply a XML file that gives a simple name to each dataset, identifies the location from which the data is served, and the mechanism of delivery. Clients like IDV can then access the catalog, and users can explore data from a variety of different locations and methods without knowing exactly where the data is coming from and how it is delivered. For the Adriatic Sea study, we made a catalog of meteorological, wave and ocean model products, all served via OPeNDAP, but some served from Hawaii, and some served from Woods Hole (Figure 5).

It is possible to use THREDDS in a more sophisticated way, setting up a THREDDS server that accepts queries to enable data searches and present collections of datasets as a single dataset for access. Often it is desirable to split the output from a long run into sequential output files because of file system constraints, and by using the aggregation capabilities of the THREDDS server, it is possible to make 30 datasets of 2GB appear to users as a single dataset of $60 \mathrm{~GB}$. THREDDS is also capable of automatically generating catalogs of locally held data, useful when the data being served is frequently updated, such as on a system serving nowcast/forecast products.

\section{Final Remarks}

We have found that making our model output CF-complaint and making it available through OPeNDAP has benefited both the community we are seeking to collaborate with and also our Adriatic MREA sea trials in a number of ways. The most important benefit has been that colleagues other than modelers have been able to explore the model fields to the full extent of their scientific interest, without being limited, say, by our choice of server-side plotting software. Not only can they explore the data just as it was generated by the model (without spatial interpolation onto rectilinear longitude/latitude grids or standard vertical levels) but they can explore the data using simple GUI-based tools like IDV, and then extract and do detailed analysis in tools they are familiar with (e.g.

Matlab). This results in much more analysis and scrutiny of the model results, and just as 
in the open-source movement where "more eyeballs on the code leads to more rapid bug fixes", has led to many helpful suggestions about potential problems with the runs and how the models themselves might be improved.

We also benefited from not having to spend time generating specialized outputs for individual collaborators. Instead of extracting just the sea surface temperature data from a model run for a remote sensing colleague, for example, we can just deliver the one or two lines of code that is necessary for them to use in their analysis environment (e.g. IDL, Matlab).

The efficiency of data extraction via OPeNDAP is a time saving benefit, but can also be essential when dealing with low-bandwidth situations, such as delivery to ships at sea. During the Adriatic Sea field trials, we conducted real-time simulations on the ship, but needed boundary conditions from a larger-scale forecast model of the Mediterranean Sea (MFSTEP, 2006). Each time interval was $24 \mathrm{MB}$ for the entire Mediterranean Sea, but because we only needed boundary conditions along the narrow southern entrance to the Adriatic Sea, we were able just to extract $0.2 \mathrm{MB}$ of information, resulting in a download of $20 \mathrm{~s}$ (instead of $40 \mathrm{~min}$ ) over our $80 \mathrm{Kbps}$ connection. One limitation with use in band-limited situations is that OPeNDAP has no provision for interrupted downloads to continue. If large datasets need to be transferred in these situations, batch-oriented methods of data retrieval with restart capability may be more desirable.

We hope that outlining this simple procedure will encourage other modelers to standardize their output by creating CF-compliant data and serving it via OPeNDAP. We also hope that it will encourage development of tools and clients that are designed to work with any CF-compliant model output, instead of only for a particular model. In this way, we can more effectively utilize the software development resources not only of the MREA community, but of the larger earth science community.

\section{Acknowledgments}

The authors thank the teams involved in projects ADRICOSM, ACE/ADRIA, and DOLCEVITA for having providing data essential for model initialization and assessment. COAMPS ${ }^{\circledR}$ is a registered trademark of the Naval Research Laboratory. S. Carniel was partially supported by the Office of Naval Research (ONR grant number N00014-05-10730). I. Janekovic was supported by the Croatian Ministry of Science, Education and Sport (grant number 0098113). 


\section{FIGURES}

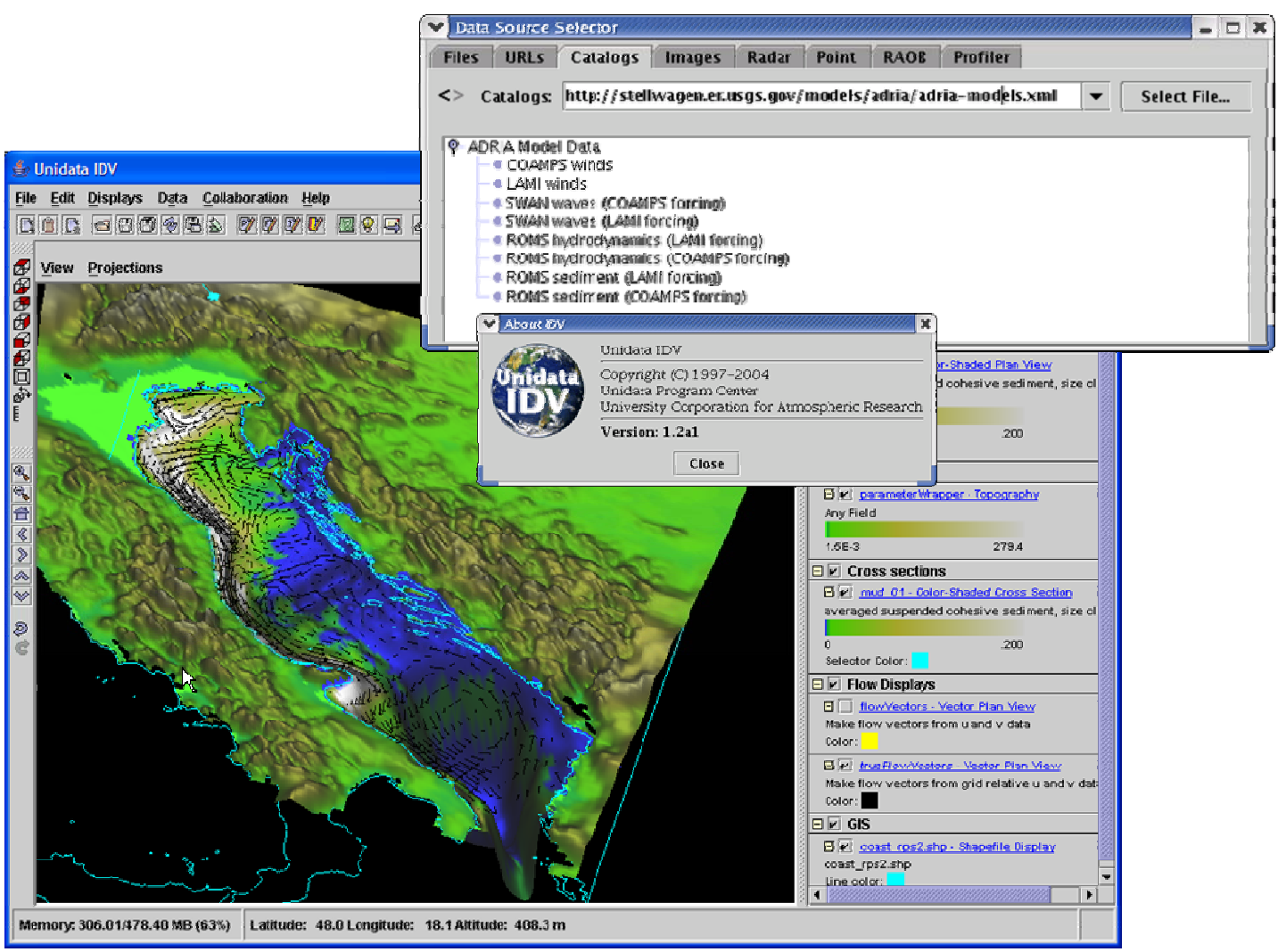

Figure 1. Example using freely available software (Unidata's Integrated Data Viewer) to browse remote model datasets from a collection of meteorological, wave, and circulation model results for the Adriatic Sea. The data sets are served by different institutions on their native model grids, and the IDV knows only that they meet the certain metadata conventions (Climate and Forecast Conventions CF1.0). 


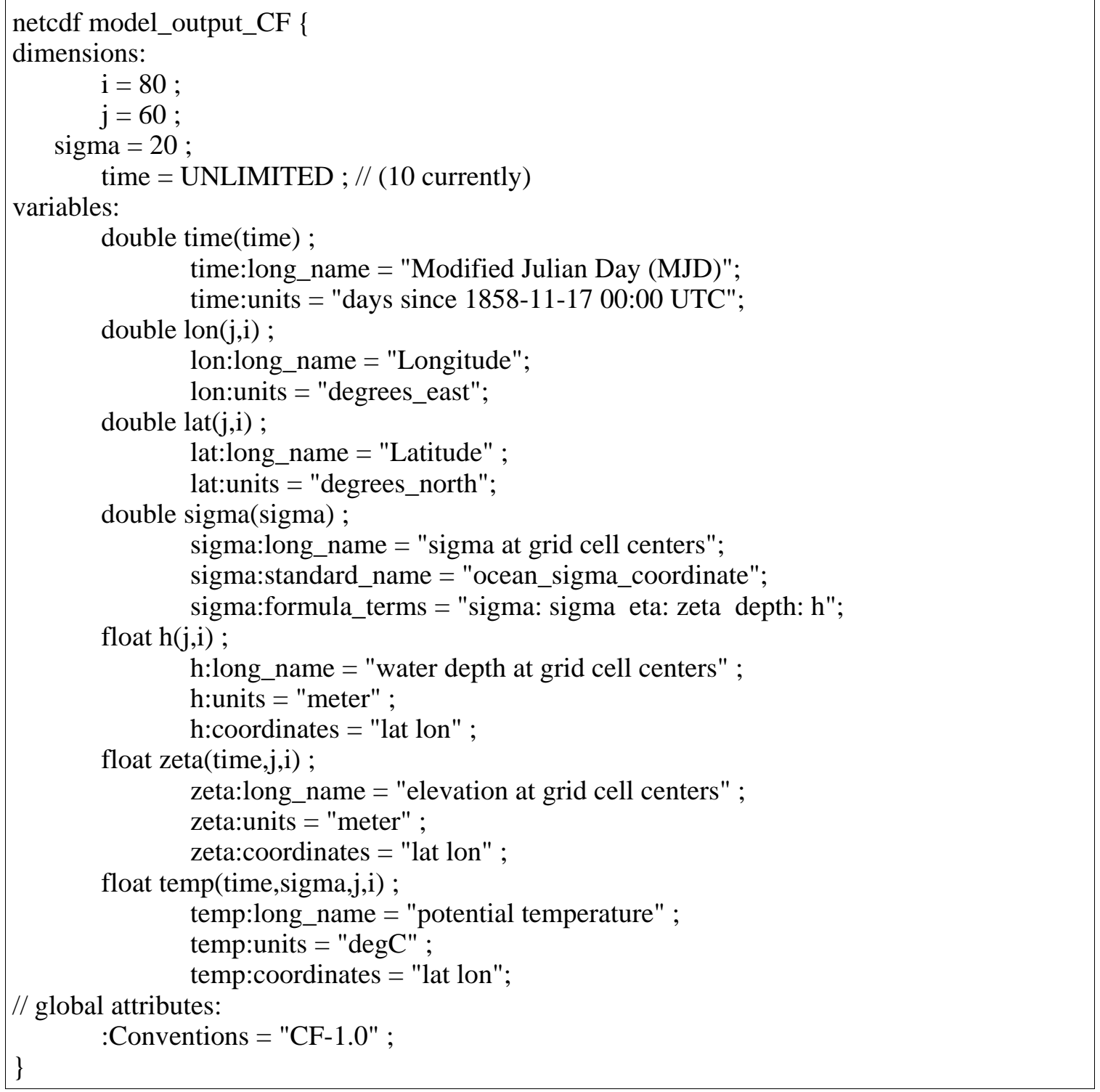

Figure 2. Example of CF-1.0 compliant output from a curvilinear, sigma coordinate ocean model. 


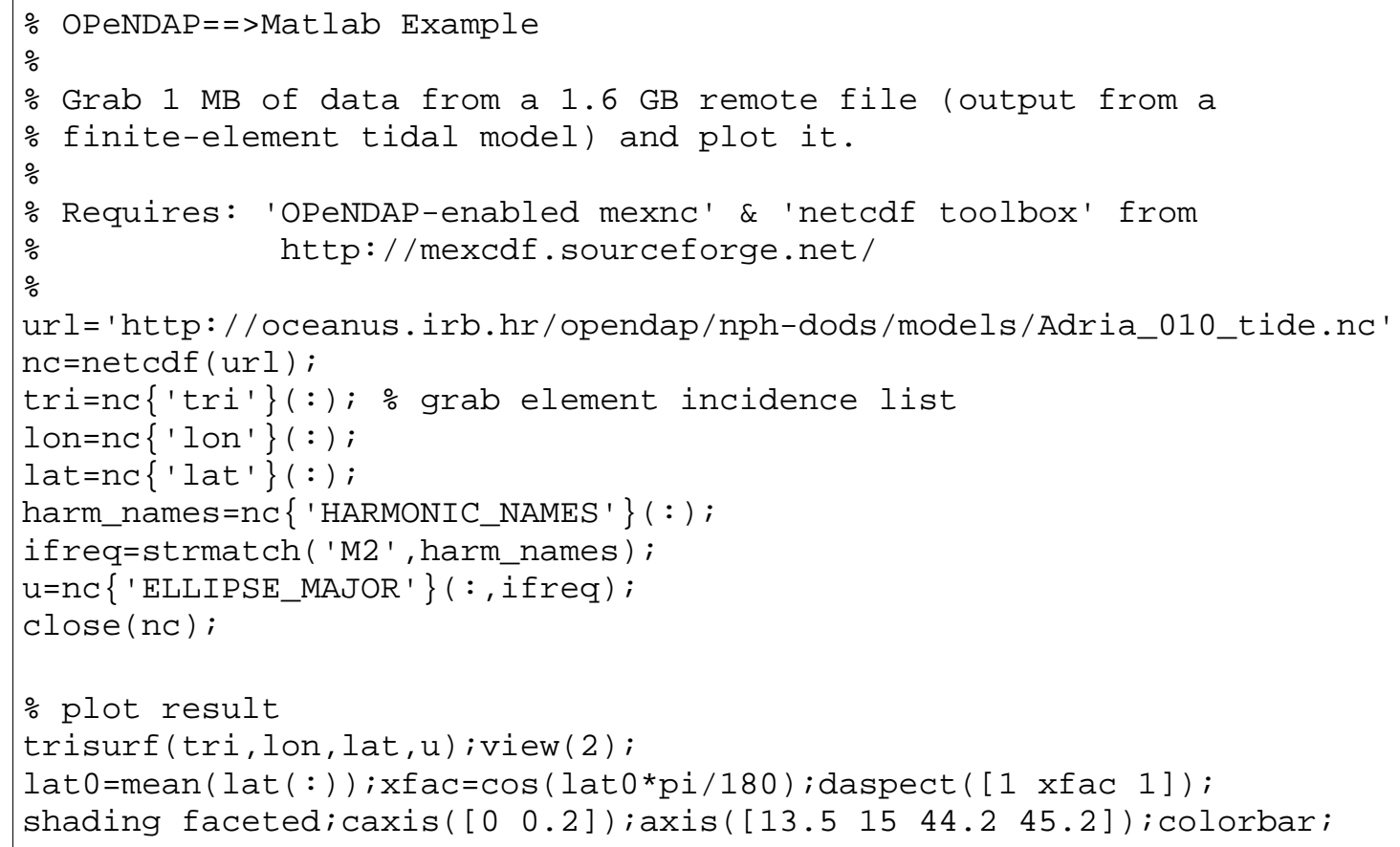

Figure 3. Sample of Matlab code to retrieve and display a field of the $\mathrm{M}_{2}$ major axis tidal current magnitude extracted from a remote 1.6 GB file using OPeNDAP. The resulting plot is shown in Figure 4. 


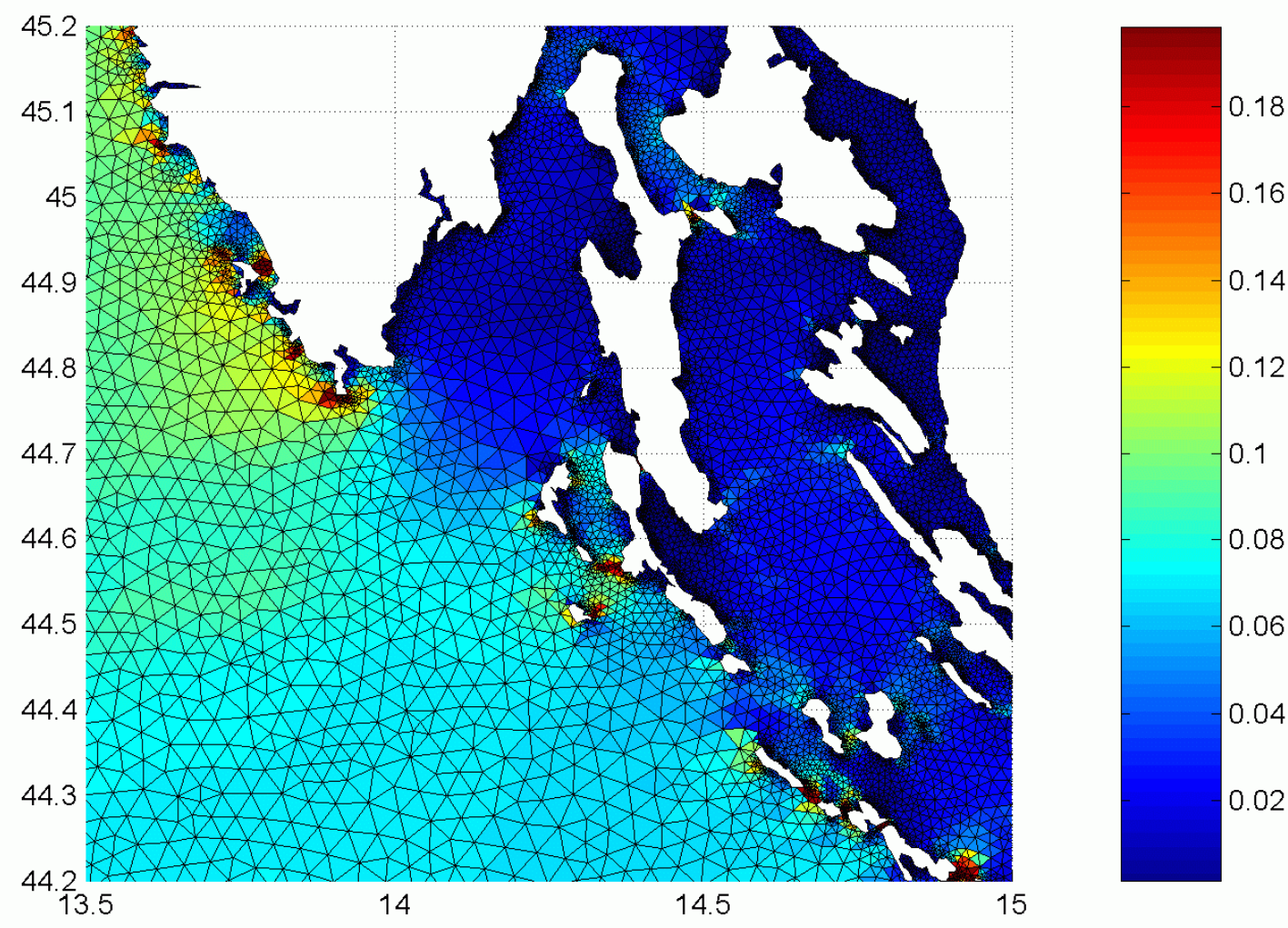

Figure 4. Snapshot of $\mathrm{M}_{2}$ major axis tidal current magnitude extracted from a remote 1.6 GB file using OPeNDAP directly into Matlab using the script shown in Figure 3. It took 14 seconds of wall clock (over a $600 \mathrm{kbps}$ DSL line) to access (and plot) the data. 


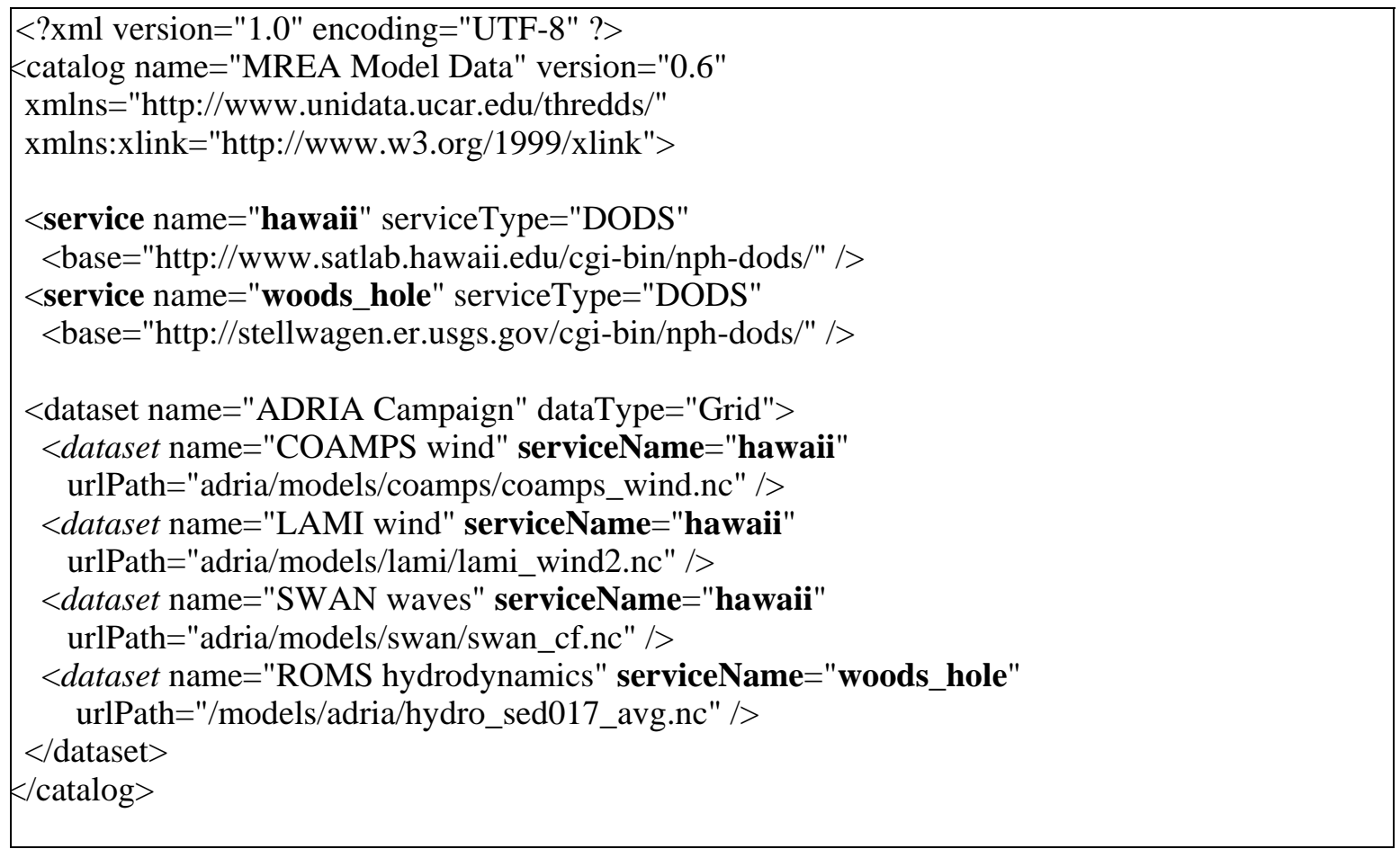

Figure 5. THREDDS catalog listing OPeNDAP datasets for wind, waves and currents served from two different locations: The University of Hawaii and the USGS Woods Hole Field Center.

\section{References}

Coelho, E., 2006. The NATO Tactical Ocean Modeling System. Journal of Marine Systems(This issue).

Eaton, B., Gregory, J., Drach, R., Taylor, K. and Hankin, S., 2003. NetCDF Climate and Forecast (CF) Metadata Conventions. <http://www.cgd.ucar.edu/cms/eaton/cfmetadata/CF-1.0.html>.

IAU, 1997. Resolution B1 of the XXXIIIrd assembly of the International Astronomical Union (IAU): On the Use of Julian Dates and Modified Julian Dates. $<$ http://www.iers.org/iers/earth/resolutions/UAI_b1.html $>$.

Janekovic, I. and Kuzmic, M., 2005. Numerical simulation of the Adriatic Sea principal tidal constituents. Annales Geophysicae, 23: 3207-3218.

Kantha, L.H., Carniel, S. and Franchi, P., 2002. Development of a real-time nowcast/forecast system for the Ligurian Sea: The GOATS-MEANS 2000 experiment. In: E. Bovio and H. Schmidt (Editors), The GOATS Joint Research Project: underwater vehicle networks for acoustic and oceanographic measurements in the littoral ocean. NATO SACLANT Centre, La Spezia, Italy, pp. 275-288.

Lee, C.M. et al., 2005. Northern Adriatic response to a wintertime bora wind event. EOS Transactions of the American Geophysical Union, 86(16): 157, 163, 165.

MFSTEP, 2006. MFSTEP web site. <http://www.bo.ingv.it/mfstep>. 
Onken, R. et al., 2005. Inter-model nesting and rapid data exchange in distributed systems. Journal of Marine Systems, 56(1-2): 45-66.

Rixen, M., 2006. Surface drift prediction in the Adriatic Sea using hyper-ensemble statistics on atmospheric, ocean and wave models: uncertainties and probability distribution areas. Journal of Marine Systems(This issue).

Sherwood, C.R. et al., 2004. Sediment dynamics in the Adriatic Sea investigated with coupled models. Oceanography, 17(4): 58-69.

Signell, R.P. et al., 2005. Assessment of wind quality for oceanographic modelling in semi-enclosed basins. Journal of Marine Systems, 50(217-233).

UNIDATA, 2006a. Integrated Data Viewer (IDV) web site. $<$ http://www.unidata.ucar.edu/software/idv/>.

UNIDATA, 2006b. NetCDF web site. <http://www.unidata.ucar.edu/packages/netcdf/> .

UNIDATA, 2006c. THREDDS web site. <http://www.unidata.ucar.edu/projects/THREDDS/>. 